\title{
Shunt-Loaded Fractal-Meandered Microstrip
}

\author{
Kimberley W. Eccleston \\ Dept of Electrical and Computer Eng., University of Canterbury, Christchurch, New Zealand \\ E-mail: kim.eccleston@elec.canterbury.ac.nz
}

\begin{abstract}
The fractal meandering approach to microstrip circuit miniaturization is limited by the need to maintain characteristic impedance. In this work we show that uniform and non-uniform shunt loading can be used yield a further level of miniaturization of Koch fractal meandered microstrips and is applied to the miniaturization of a $1 \mathrm{GHz}$ bandstop filter. The miniaturized filter has an area $11 \%$ of the area of a filter using straight microstrips alone, and $57 \%$ of the area of a filter miniaturized by second order fractal meandering alone.
\end{abstract}

\section{INTRODUCTION}

Transmission line circuits underscores microwave technology offering realization opportunities unavailable to low frequency circuit technology. Some circuit examples that exploit transmission line include branchline couplers, rat-race couplers, and transmission line filters. The physical length of transmission lines are inversely proportional to frequency so transmission line circuits operating at low microwave frequencies tend to be large.

Often transmission lines are realized with microstrips on a printed circuit board (PCB) or on a semiconductor substrate. Circuits comprising microstrip elements, such as branchline and rat-race couplers, tend to leave large areas of the printed circuit board interior to the coupler unoccupied.

Meandering of the microstrip is often used to make better use of PCB area. Meandered microstrips generally have a rectangular boundary which may be sub-optimal in applications employing several microstrip sections. For example, a triangular or a sectoral shaped boundary, are a better choice for branchline and rat-race couplers [1].

Slow-wave microstrip structures can achieve a given electrical length with a shorter structure as well as occupy PCB area interior to rat-race and branchline couplers [2]-[5]. A combination of meandering of slow-wave structures [6][7], and fractal meandering of microstrips [8][9] has also been applied to achieve compact microstrip structures.

Coupling between parallel microstrips limits the extent of conventional meandering. Fractal meandering has the advantage of separating parallel microstrips. Another advantage of fractal meandering is that the boundary geometry is maintained with each iteration [8][9]. A true fractal involves an infinite number of iterations, but when applied to microstrip, at most two iterations can be achieved [8][9]. It is therefore the purpose of this work to investigate further size reductions in a

fractal meander using periodic shunt loading. Section II discusses the challenges facing fractal meandering of microstrips, Section III considers periodic loading of the fractal microstrip and Section IV applies the proposed Fractal microstrip to a bandstop filter [10].

\section{Fractal MEANDERING Challenges}

We consider the Koch fractal [9] as it has a triangular boundary which makes it attractive for the square shaped bandstop filter [10]. Fig. 1 shows the initiator (a straight microstrip), and the first two Koch fractal meander iterations. In all three cases, the microstrips have the same electrical length and characteristic impedance. Essentially, at each iteration, all the straight microstrip sections are divided into five equal lengths, which are configured as shown in Fig. 1 (b) The resulting triangular boundary is apparent which makes it an elegant component in a branch-line coupler [9], or a bandstop filter [10], employing four $90^{\circ}$ microstrips forming a loop. Ideally, the dimensions and area of a triangular boundary are respectively $60 \%$ and $36 \%$ of the triangular boundary of the previous iteration.

However, unlike theoretical fractals, the width of the microstrip must remain constant to maintain the required characteristic impedance. It is apparent from Fig. 1(c) that further iterations are not possible as the lengths of the straight elements are equal in size to the bends. This problem is exacerbated by the need to reduce microstrip length to compensate for the additional phase shift caused by bends introduced at each iteration [9].

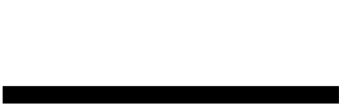

(a)

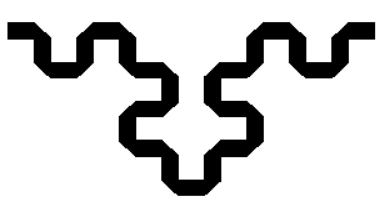

(c)

Fig. 1. Koch fractal meandered microstrips with the same electrical lengths: (a) zeroth order or initiator, (b) first iteration and (c) second iteration 
It is also clear from Fig. 1(c) that the width of the microstrip dominates meander boundary dimensions at higher iterations thereby restricting the amount of size reduction achieved at each iteration.

Realizations thus far reported operated at $2.4 \mathrm{GHz}$ where a second iteration was made possible by either considering high impedance (hence narrow) microstrips [8] or using a very thin substrate thereby reducing microstrip width proportionally [9]. The challenge is to consider realization of normal values of characteristic impedance (eg. $50 \Omega$ ) and use a practical substrate thickness.

We consider a $50 \Omega$ microstrip whose electrical length is $90^{\circ}$ at $1 \mathrm{GHz}$ and is realized on a $0.254 \mathrm{~mm}$ thick substrate whose dielectric constant is 2.22 . The width and length of a straight microstrip to achieve this is $0.76 \mathrm{~mm}$ and $54.7 \mathrm{~mm}$ respectively. A bandstop filter [10] constructed from such straight microstrips would have an area of $3075 \mathrm{~mm}^{2}$. Although a second order fractal iteration is possible (including compensation for bend phase shifts), a third iteration is unachievable. Never-the-less, the longest dimension of the second order fractal meander is $21.9 \mathrm{~mm}$ or $40 \%$ of the original straight microstrip length. This is to be compared with an ideal second order fractal which would have a length $36 \%$ of the initiator. The area of a bandstop filter [10] using the second order fractal meander would be $571 \mathrm{~mm}^{2}$ which is $19 \%$ of the area of a filter using straight microstrips alone. The challenge is to further reduce the circuit size.

\section{SHUNT-LOADED FRACTAL-MEANDER}

It is well known that periodic shunt loading of transmission lines results in shorter structures to achieve a given electrical length [2]-[5]. Another important benefit of periodic shunt loading is that the microstrip width can be reduced to maintain a certain characteristic impedance [11], and this is useful to address the above mentioned fractal meander restrictions due to microstrip width.

A number of loading geometries were considered. Fig. 2 shows shunt loading of each straight element of the second order fractal meander. In both case depicted in Fig. 2 the triangular boundary is maintained. With loading on one side (Fig. 2(a)), the horizontal length is $20.4 \mathrm{~mm}$ and microstrip width is $0.67 \mathrm{~mm}$. With loading on both sides (Fig. 2(b), the horizontal length is $19.1 \mathrm{~mm}$ and the microstrip width is 0.37 $\mathrm{mm}$. Compared to the second order fractal, a size reduction of $13 \%$ has been achieved for the structure of Fig. 2(b).

The second order fractal structures of Fig. 2 comprise five first order sub-fractals whose electrical lengths are $18^{\circ}$. Each of these first order fractals has unoccupied area in its loop. Therefore, the middle stub of each first order fractal was extended to increase loading of each $18^{\circ}$ first order fractal. Fig. 3 shows a resulting second order fractal and its horizontal length is $17.9 \mathrm{~mm}$ (representing an $18 \%$ size reduction compared to the second order fractal meander) and the microstrip width is $0.36 \mathrm{~mm}$. Non-uniform loading in conjunction with uniform periodic loading was also considered to fill unoccupied $\mathrm{PCB}$ area. Fig. 4 shows the resulting structure and its horizontal length is $16.3 \mathrm{~mm}$ (26\% size reduction compared to the second order fractal meander) and the microstrip width is $0.22 \mathrm{~mm}$. This level of size reduction is comparable to that ideally achievable with an extra fractal iteration. Moreover, the geometries of Fig. 2 and Fig. 3 look similar to third order fractal iterations when viewed from a distance.

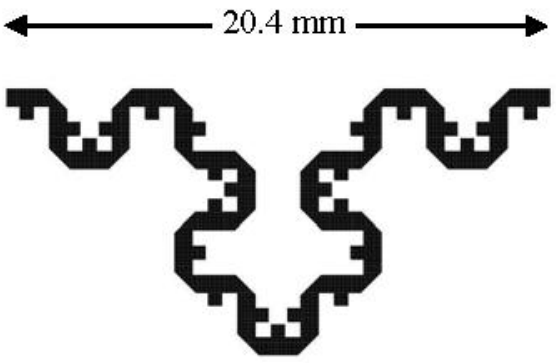

(a)

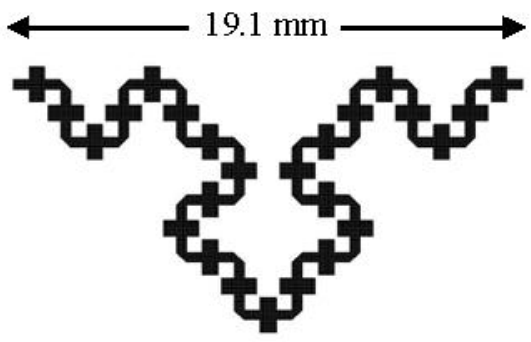

(b)

Fig. 2. Uniform periodic loading of the second order meander: (a) loading on one side, and (b) loading on both sides

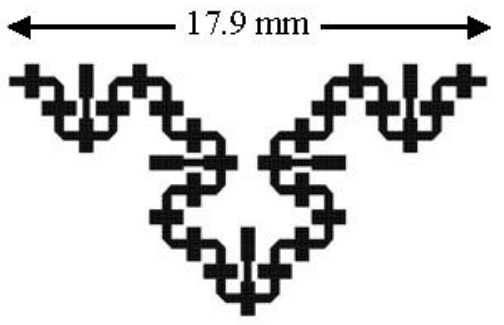

Fig. 3. Second order fractal with two levels of uniform loading

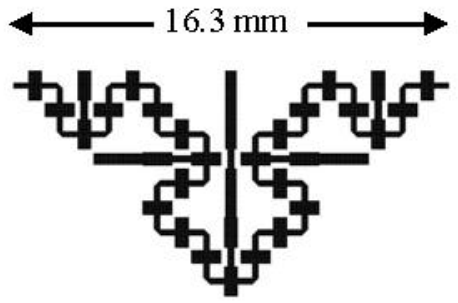

Fig. 4. Second order fractal with non-uniform and two levels of uniform loading 
The loaded fractals of Fig. 2 - Fig. 4 were designed with the aid of the "fine-tune" tool of Microwave Office ${ }^{\mathrm{TM}}$ [12]. Only the main microstrip width and section lengths were the independent (and hence tuned) variables. The stubs lengths and widths were all related to the main microstrip width and length to ensure geometric constraints were satisfied. The objective of tuning was to achieve minimum reflection (reference to $50 \Omega$ ) and $90^{\circ}$ insertion phase. During the design phase, the circuit simulator was used for computation speed.

Due to the close proximity of microstrip discontinuities and the close proximity of microstrip elements, full-wave electromagnetic (EM) simulations were also performed. The cell size was $0.04 \mathrm{~mm} \times 0.04 \mathrm{~mm}$ during the EM simulations which is extremely small compared to the wavelength but necessarily small to accurately resolve the fine details of the structures. Fig. 5 shows both the circuit and EM simulation results for the structures shown in Fig. 3 and Fig. 4. The EM simulated phase is not shown as it was nearly identical to the circuit simulations. Based upon the EM simulation results, the actual characteristic impedance is estimated to be $58 \Omega$ and 60 $\Omega$ for the structures of Fig. 3 and Fig. 4 respectively.

The smallest spacing between separation between parallel copper track edges for either of the meanders of Fig. 3 or Fig. 4 is $0.24 \mathrm{~mm}$. This is well within the capability of PCB router technology [13].

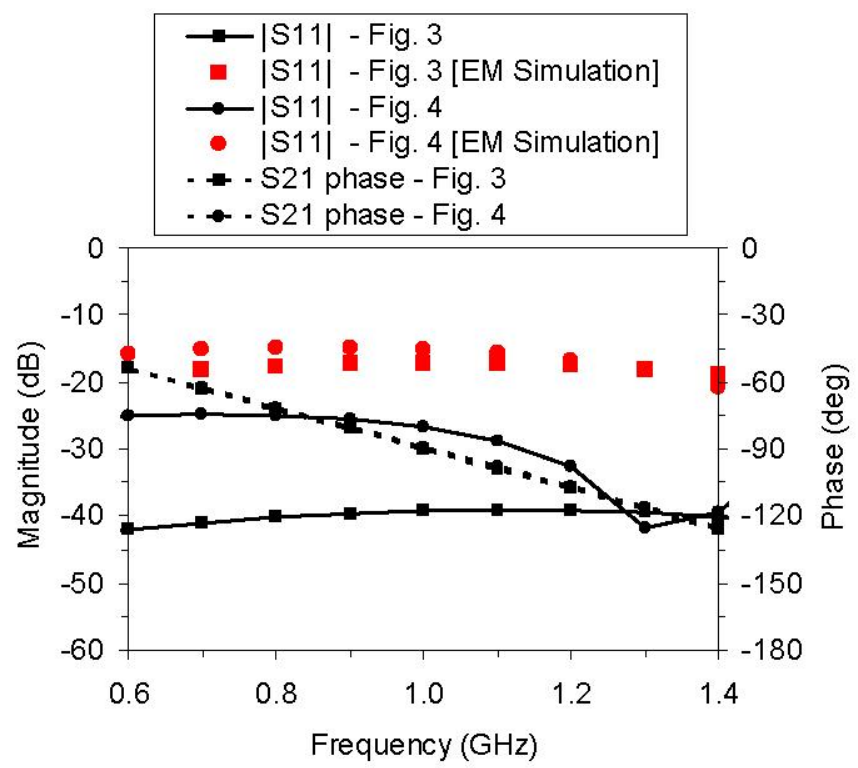

Fig. 5. Simulated S-parameter frequency responses of the structures shown in Fig. 3 and Fig. 4

\section{COMPACT BAND-STOP FILTER}

A bandstop filter can be constructed with a microstrip loop whose circumference is one guide-wavelength at the stop frequency and whose ports are located one-quarter guidewavelength apart on the loop [10]. Fig. 6 shows this type of filter realized using the meandered microstrips of Fig. 3 and Fig. 4. The filters of Fig. 6(a) and Fig. 6(b) occupy an area of $400 \mathrm{~mm}^{2}$ and $330 \mathrm{~mm}^{2}$ respectively. This means that the filter of Fig. 6(b) occupies $11 \%$ of the area of a filter using straight microstrips alone or $57 \%$ of the area of a filter using the second order fractal meanders. This is to be compared to an ideal third order Koch fractal which would have an area of $5 \%$ of the area of the square initiator and $36 \%$ of the second order fractal.

Circuit simulations of the two filters in Fig. 6 were performed and the results are shown in Fig. 7. EM simulation of the complete filters was not possible due to the computation time. Rather, EM simulation results for the fractals of Fig. 3 and Fig. 4 were combined with a few extra circuit elements (eg. bends and short microstrips to connect the four fractal meanders) to obtain the EM simulation results shown in Fig. 7. The EM results differ only slightly from the circuit simulation results. This outcome stems from the insensitivity filter to characteristic impedance; rather it is the electrical length that is critical.

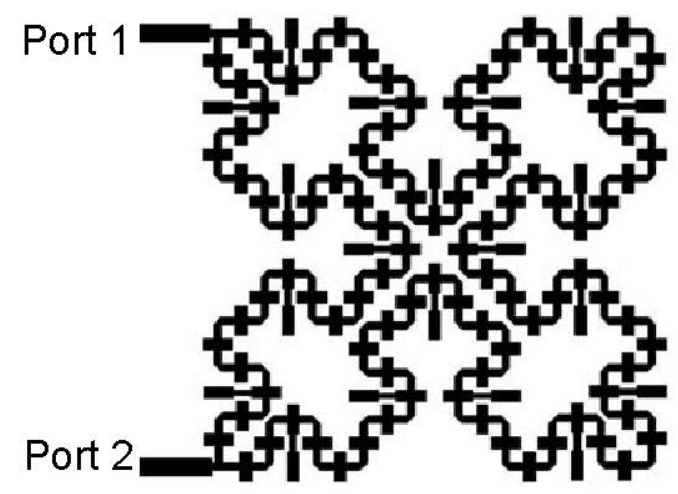

(a)

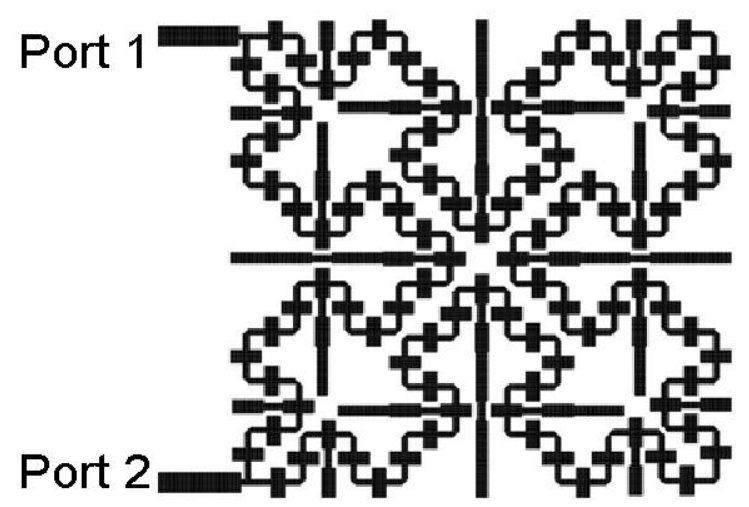

(b)

Fig. 6. Bandstop filters using four fractal meanders of the type shown in: (a) Fig. 3, and (b) Fig. 4

These simulations ignore spurious coupling between the four meanders. However, the very nature of the fractal 
geometry means that most elements of one meander are separated by a significant distance from elements of the other meanders. The exception is that 6 bends of each meander are about $0.3 \mathrm{~mm}$ (compared with the substrate thickness of 0.254 $\mathrm{mm}$ ) from corresponding bends of adjacent meanders. EM simulations of a pair of bends in such close proximity show that the coupling is very small and therefore the EM simulation results shown in Fig. 7 are representative.

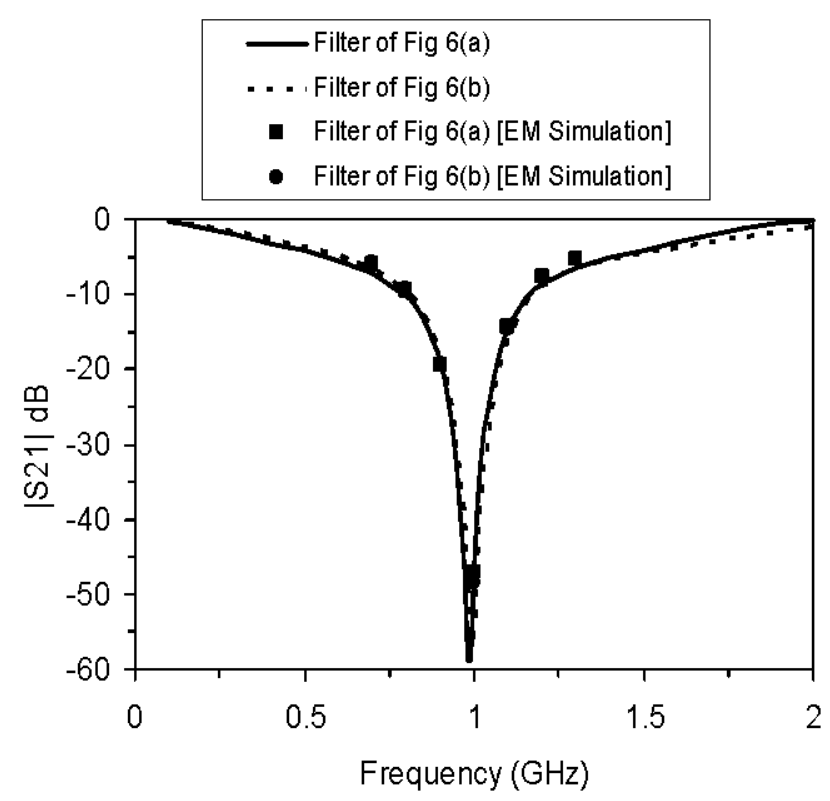

Fig. 7. Simulated response of the filters shown in Fig. 6

\section{CONCLUSION}

In this paper we have shown that both uniform periodic and non-uniform shunt loading of fractal meandered microstrips results in significant reduction of size. The amount of size reduction is comparable to that achieved by a fractal iteration. The resulting structures could be manufactured using PCB router technology. The shunt loading was achieved by constant width microstrip sections and the stubs partially fill otherwise unoccupied PCB area. There still remains unutilized PCB area and this could be utilizing by using stub geometries similar to the void geometries.

\section{REFERENCES}

[1] M. H. Awida, A. M. E. Safwat and H. El-Hennawy, "Compact Rat-Race Hybrid Coupler Using Meander Space-Filling Curves", Microwave and Optical Technology Letters, vol. 48, no. 3, pp 606-609, March 2006.

[2] J. Wang, B.-Z Wang, Y.-X. Guo, L. C. Ong and S. Xiao, "Compact slow-wave microstrip rat-race ring coupler", Electronics Letters, vol. 43. no. 2, pp $111-112$, 18 Jan 2007 .

[3] J. Wang, B.-Z. Wang, Y.-X. Guo, L. C. Ong and S. Xiao, "A Compact Slow-Wave Microstrip Branch-Line Coupler With High Performance", IEEE Microwave and Wireless Components Letters, vol. 17 , no. $7, \mathrm{pp}$ $501-503$, July 2007
[4] J.-T. Kuo, J.-S. Wu and Y.-C. Chiou, "Miniaturized Rat Race Coupler With Suppression of Spurious Passband", IEEE Microwave and Wireless Components Letters, vol. 17, no. 1, pp 46-48, Jan 2007.

[5] S.-S. Liao, P.-T. Sun, N.-C. Chin and J.-T. Peng, "A Novel CompactSize Branch-Line Coupler", IEEE Microwave and Wireless Components Letters, vol. 15 , no. 9, pp 588-590, Sept 2005.

[6] D. Nesic, "Slow-wave EBG microstrip rat-race hybrid ring", Electronics Letters, vol. 41, no. 21, pp $1181-1183,13$ Oct 2005.

[7] C.-W. Tang and M.-G. Chen, "Synthesizing Microstrip Branch-Line Couplers With Predetermined Compact Size and Bandwidth", IEEE Trans. on Microwave Theory and Techniques, vol. 55, no. 9, $1926-$ 1934, Sept 2007.

[8] H. Ghali and T. A. Moselhy, "Miniaturized Fractal Rat-Race, BranchLine and Coupled-Line Hybrids", IEEE Trans. on Microwave Theory and Techniques, vol. 52, no. 11, 2513-2520, Nov. 2004.

[9] W.-L. Chen and G.-M. Wang, "Design of Novel Miniaturized FractalShaped Branch-Line Couplers", 2007 Asia-Pacific Microwave Conference, pp $2155-2158$.

[10] L.-H. Hsieh and K. Chang, "Compact, Low Insertion-Loss, SharpRejection, and Wide-Band Microstrip Bandpass Filters", IEEE Trans. on Microwave Theory and Techniques, vol. 51, no. 4, pp 1241 - 1245, April 2003.

[11] K. W. Eccleston and H. M. Ong, "Compact Planar Microstripline Branch-Line and Rat-Race Couplers", IEEE Transactions on Microwave Theory and Techniques, Vol 51, No. 10, pp 2119-2125, Oct 2003.

[12] Microwave OfficeTM, Applied Wave Research: http://web.awrcorp.com/

[13] LPKF Laser \& Electronics AG. http://www.lpkf.com/ 